Teja Fabjan, Eda Vrtačnik-Bokal, Kristina Kumer and Joško Osredkar*

\title{
Determination of oxidative stress balance in follicular fluid
}

https://doi.org/10.1515/labmed-2017-0106

Received August 18, 2017; accepted January 23, 2018

\section{Abstract}

Background: The role of oxidative stress in female reproduction is an area that needs more investigation. This study aims to assess the oxidative stress levels in follicular fluid (FF) samples of women undergoing in vitro fertilization (IVF) and to relate them to different diagnoses.

Methods: A total of 199 woman were enrolled; 37 of them were diagnosed with polycystic ovary syndrome (PCOS), 71 with endometriosis and 41 with tubal factor infertility (TFI) and 50 of the patients were used as a control group. A sample of FF was collected from each patient at an oocyte retrieval day and analyzed for oxidative stress by measuring a class of reactive oxygen metabolites (ROMs) (dROMs test) and also analyzed for the plasma antioxidant test (PAT).

Results: The data analyzed in FF were related to different diagnoses. Groups were not significantly different in age and body mass index (BMI), except for the PCOS group. There was a significant difference between dROMs and PAT levels in FF of patients vs. control group. The same finding was seen when the dROM/PAT ratio was used.

Conclusions: We conclude that the evaluation of oxidative stress in FF needs more investigation with regard to markers in the follicular microenvironment.

Keywords: endometriosis; female infertility; follicular fluid; FRAS5; IVF; oxidative stress; PCOS; tubal factor infertility.

*Correspondence: Professor Joško Osredkar, PhD, University Medical Centre Ljubljana, Institute of Clinical Chemistry and Biochemistry, Njegoševa 004, 1000 Ljubljana, Slovenia, E-Mail: josko.osredkar@kclj.si

Teja Fabjan and Kristina Kumer: University Medical Centre Ljubljana, Institute of Clinical Chemistry and Biochemistry, Ljubljana, Slovenia Eda Vrtačnik-Bokal: University Medical Centre Ljubljana, Division of Gynaecology, Department of Human Reproduction, Ljubljana, Slovenia
Introduction

The human organism is exposed to various forms of stress (physical, psychological or chemical). A certain amount of stress is always present and in some way directs, promotes or inhibits the functioning of the human body. Oxidative stress is a kind of "chemical stress" that is present in living organisms due to an increased quantity of potentially harmful agents, reactive oxygen species (ROS). By definition, oxidative stress is a direct consequence of the harmful action of an increased quantity of these highly reactive substances on cells and tissue in the body. In humans, the production of ROS takes place in every cell of the body. Oxygen is indispensable for life - the basis of the breathing process - but at the same time many ROS are produced. These include not only free oxygen radicals, such as hydroxyl and superoxide radicals, but also other compounds such as hydrogen peroxide and ozone.

Particularly important among these reactive species (RS) are the hydroperoxides, formed from different sources (lipid, protein, DNA). They can directly oxidize substrates containing SH groups (thiols) or trigger the propagation of the oxidative process. In a normal physiological condition, we have an equilibrium between ROS (RS) production and the protective and the complex antioxidative (AO) system [1-3].

It seems that despite their high chemical reactivity, most generated ROS do not lead to serious negative physiological consequences for health. This is mainly due to the action of highly efficient systems of ROS neutralization operating in concert with reparation and elimination of ROS-modified molecules; this always exists in what may be called the basal steady-state (stationary) level $[4,5]$.

Human follicle development involves multiple intraovarian and endocrine interactions that create a changing intrafollicular microenvironment for optimal oocyte development [6]. The impact of oxidative stress in follicular fluid (FF) on oocyte maturation, fertilization and implantation of embryos has received considerable attention during the recent years [7].

FF is the net result of both the transfer of plasma constituents to follicles and the secretory activity of granulose and theca cells. The oocyte develops within the FF environment and this intimately affects the quality of oocytes and 
their interaction with sperm, thus affecting implantation and embryonic development [8]. Oxidative stress contributes to oocyte quality, and its degree can be assessed by biomarkers of lipid peroxidation [9]. The effects of oxidative stress may be further altered by environmental factors.

In this study, we considered patients with three different infertility diagnoses. Polycystic ovary syndrome (PCOS) is the most frequent endocrine abnormality in women during reproductive age, with a prevalence of nearly $5-10 \%$. PCOS is the main reason for an ovulatory infertility [10]. The recent reports demonstrate that ovarian dysfunction in PCOS results from ovarian follicle disorders in two ways [11, 12]. First, early follicular development is excessive, so women with PCOS are characterized by the development of an increased number of small antral follicles (two- to three-fold that of normal ovaries) and second, the selection of the dominant follicle from the excessive pool of selectable follicles does not occur.

Endometriosis is a complex estrogen-dependent inflammatory disease that is defined as the presence of endometrial glands and stroma-like tissue outside the uterine cavity and musculature [13, 14]. Ectopic endometrial tissue can be found in different parts of the peritoneal cavity and thus can form three types of endometriosis with different etiologies and pathogenesis: peritoneal, ovarian and deep-infiltrating endometriosis [15-18]. Multiple lines of scientific evidence support Sampson's widely accepted theory of retrograde menstruation where fragments of eutopic endometrium pass backward through the fallopian tubes into the peritoneal cavity during menstruation and persist there due to an impaired immune system $[19,20]$.

Tubal factor infertility (TFI) is a disorder in which the fallopian tubes are blocked or damaged. Scar tissue, infections and tubal ligation are often causes of tubal disease. Scar tissue resulting from endometriosis or abdominal or gynecological surgery such as bowel surgery, cesarean section or a ruptured appendix can block an egg from entering or traveling down the fallopian tube to meet the sperm, thus preventing fertilization [21, 22].

In our study we aimed to evaluate the impact of oxidative stress in presented clinical conditions associated with infertility by measuring reactive oxygen metabolites (ROMs) antioxidants in the FF of the recruited patients.

\section{Materials and methods}

\section{Participants}

This observational study was carried out between March 2013 and April 2014 at the University Medical Centre Ljubljana, Reproductive Medicine Unit. A total of 199 White European women, reporting different factors of infertility for in vitro fertilization (IVF), were included: 37 were diagnosed with PCOS, 71 with endometriosis and 41 with TFI and 50 were selected as a control group, where infertility was a consequence of male infertility. Basic characteristics of the patient groups and healthy comparison group are presented in Table 1. All patients signed an informed consent. Ethical approval was obtained from the Slovenian National Committee on Medical Ethics.

\section{Samples collection}

A sample of FF was collected from each patient on the oocyte retrieval day and analyzed for oxidative stress by measuring a class of ROMs (dROMs test) and for the plasma antioxidant capacity (PAT test) on FRAS5 system (H\&D srl, 43124 Parma, Italy). Both methods were verified for FF. All women underwent controlled ovarian stimulation. During the oocyte pick-up at the $36^{\text {th }}$ hour following recombinant human chorionic gonadotropin (hCG) administration (when the largest follicle was $18 \mathrm{~mm}$ in diameter), the collection of the FF samples from each

Table 1: Basic patient characteristics.

\begin{tabular}{lrrrr}
\hline & $\begin{array}{r}\text { Endometriosis } \\
(\mathbf{9 5 \%} \text { Cl for the mean) }\end{array}$ & $\begin{array}{r}\text { PCOS } \\
(\mathbf{9 5 \%} \text { Cl for the mean) }\end{array}$ & $\begin{array}{r}\text { Tubal factor infertility } \\
\mathbf{9 5 \%} \text { Cl for the mean) }\end{array}$ & $\begin{array}{r}\text { Healthy control (95\% } \\
\mathbf{C l} \text { for the mean) }\end{array}$ \\
\hline $\mathrm{n}$ & 71 & 37 & 41 & 50 \\
Age, years & $33.8(33.1-34.5)$ & $30.8(29.4-32.2)$ & $32.8(31.6-34.1)$ & $31.62(30.5-32.7)$ \\
Height, cm & $165.9(164.3-167.5)$ & $164.8(162.8-166.7)$ & $167.5(165.8-169.1)$ & $167.4(165.9-169.1)$ \\
Weight, kg & $60.3(58.5-62.0)$ & $70.7(65.1-76.3)$ & $64.4(61.2-67.8)$ & $63.4(61.1-65.8)$ \\
BMl & $21.6(21.2-22.3)$ & $26.1(24-28.1)$ & $22.4(21.1-24.1)$ & $22.2(21.3-23.3)$ \\
Oocytes retrieved & $6.5(5.3-7.8)$ & $11.2(8.5-13.9)$ & $9.0(7.1-10.9)$ & $9.3(7.5-11.1)$ \\
Pregnancy rate, \% & 38.9 & 39.5 & 48.8 & 60.0 \\
\hline
\end{tabular}

$\mathrm{Cl}$, confidence interval. 
patient was performed by careful aspiration through the vagina under ultrasound guidance. In the procedure selected the first leading follicle, which contained oocytes at metaphase II, by using follicle flushing. Moreover, only FF samples without blood clot were used for the measurements to minimize any possible interference with the photometric assay. The FF samples collected were centrifuged at $3000 \times \mathrm{g}$ for $10 \mathrm{~min}$ (to precipitate blood cells and to remove cellular components). All supernatants were stored at $-80{ }^{\circ} \mathrm{C}$ until assayed.

\section{Sample analysis}

The most reliable way to assess oxidative stress would be direct determination of the level of free radicals, but the very short lifetime of these species and the intrinsic difficulty of routine quantitative electron paramagnetic resonance (EPR) determinations - let alone the cost of EPR spectrometers themselves - make this an impracticable method for diagnostic laboratories.

The dROMs test is a photometric test commonly used as a specific method for determining hydroperoxides in plasma samples. Hydroperoxides are potentially oxidative agents generated in cells by RS attack, particularly when directed at lipids and also other biochemical substrates such as amino acids, peptides, proteins and nucleotides.

Antioxidants represent the main barrier of defense against damaging aggression due to RS, in particular by ROS. Antioxidants can derive from either endogenous or exogenous agents and they counterbalance ROS production (in particular hydroperoxides). The plasma $\mathrm{AO}$ capacity is a measure of physiological, environmental and nutritional factors (exposure to ROS and antioxidant supplementation) determining the redox status in humans, and it can underline the oxidative stress conditions. A higher level of ROS and a consequent lower level of antioxidants lead to a condition of oxidative stress, an emerging risk factor for the development of many diseases (inflammatory, infectious and degenerative disease). Moreover, changes in the AO plasma content after supplementation may provide information on the absorption and bioavailability of nutritional compounds and efficacy of AO therapy $[23,24]$.

The dROMs test is the only commercially available test that is precise and accurate for completely evaluating the harmful and pro-oxidant components and the oxidative stress in particular for redox inflammatory reactions. The formation of hydroperoxides is considered to be the most important consequence of peroxidation induced by free radicals in a biological fluid; therefore, the test has been designed to monitor the oxidation of the colorless chromogen to its red radical cation by the alkoxyl and alkylperoxyl radicals originating from the reaction of the hydroperoxides with the iron (II) ions released in the acidic medium. ROMs in the presence of iron are able to generate alkoxyl and peroxyl radicals (Fenton's reaction). Such radicals are able to oxidize an alkyl-substituted aromatic amine, thus transforming them into a pink-colored derivative, photometrically quantified at 505-546 nm. As a hydroperoxide detector, the test uses N,N-diethyl-pphenylenediamine (DEPPD); when added to a sample (FF) this dissolved beforehand in an acid buffer (acetic acid/ sodium acetate $\mathrm{pH}$ 4.8). The chromatic changes depend exclusively on DEPPD oxidation; one electron yielded from the neutral aromatic ammine form (colorless) to the radicalic species (generated in the biological sample by lowered $\mathrm{pH}$ ) and the cation-radical of DEPPD (pink color) was formed. The intensity of color is directly proportional to the concentration of ROMs [25]. We followed the instructions for measuring on FRAS5: $20 \mu \mathrm{L}$ of the samples are transferred and mixed gently into the cuvette containing $\mathrm{R} 2$ reagent, previously posed in the thermostated housing. Twenty microliters of R1 reagent are added to the cuvette, properly mixed by inversion and put in the opposite reading cell, for providing the results which are directly expressed as Carr.U. (1 Carr.U. $=0.08 \mathrm{mg} / \mathrm{dL} \mathrm{H}_{2} \mathrm{O}_{2}$ ) [26-28].

The PAT is used to quantify the water-soluble antioxidant contents in biological fluids by measuring sample ability of reducing iron from ferric ion $\left(\mathrm{Fe}^{3+}\right)$ to ferrous ion $\left(\mathrm{Fe}^{2+}\right)$. The quantified antioxidant potential is attributable to the major component of the plasma barrier to oxidation (vitamin C, vitamin E, uric acid, bilirubin). Samples are dissolved in a colored solution previously obtained by mixing a source of ferric ions $\left(\mathrm{FeCl}_{3}\right.$, ferric chloride, $\mathrm{R} 2$ reagent) with a special chromogenic substrate (a thiocyanate derived compound, R1 reagent). A $50 \mu \mathrm{L}$ measure of reagent $\mathrm{R} 2$ is transferred and mixed gently in the cuvette containing the reagent $\mathrm{R} 1$, seated in the opposite reading cell as the reagent blank; $10 \mu \mathrm{L}$ of the sample are added in the same cuvette and mixed by inversion. After $5 \mathrm{~min}$ of incubation at $37^{\circ} \mathrm{C}$, the instrument provides the result as $\mu \mathrm{mol} / \mathrm{L}$ of antioxidant substances. Prevalent and stable species of $\mathrm{Fe}(\mathrm{SCN})_{3}$ are formed. The characteristic of this species is the red-brown color that is detectable at $505 \mathrm{~nm}$. Results of the PAT test performed on the FRAS5 analytical photometric system are expressed in U.Cor, where 1 U.Cor correspond to $\mu \mathrm{mol} / \mathrm{L}$ of vitamin C obtained with the formula: PAT value $\times 1.4)[23,24,29]$.

The performance of both tests on the FRAS5 analyzer is presented in Table 2 [30]. 
Table 2: Quality control data of dROM and PAT tests.

\begin{tabular}{|c|c|c|c|c|c|c|}
\hline \multirow{2}{*}{$\frac{\text { Characteristic }}{\text { Linearity }}$} & \multicolumn{3}{|r|}{$\mathrm{dROM}^{\mathrm{a}}$} & \multicolumn{3}{|c|}{ PAT test ${ }^{\mathrm{b}}$} \\
\hline & & $50-50$ & Carr.U. & & -6000 & $\begin{array}{l}\text { ol/L of } \\
\operatorname{amin} C\end{array}$ \\
\hline \multirow[t]{2}{*}{ Normal range } & \multicolumn{3}{|c|}{ 250-300 Carr.U. } & \multicolumn{3}{|c|}{ 2200-2800 U.Cor } \\
\hline & n & Mean & CV, \% & $n$ & Mean & CV, \% \\
\hline Within-run & 10 & 255 & 3.91 & 10 & 1975 & 1.06 \\
\hline Between-run & 8 & 314 & 10 & 16 & 2286 & 4.17 \\
\hline
\end{tabular}

$\mathrm{CV}$, coefficient of variation. ${ }^{\mathrm{a}} 1$ Carr.U. $=0.08 \mathrm{mg} / \mathrm{dL} \mathrm{H}_{2} \mathrm{O}_{2} \cdot{ }^{\mathrm{b}} 1$ U.Cor $=1.4 \mu \mathrm{mol} / \mathrm{L}$ of ascorbic acid.

\section{Statistical analysis}

Data were described as number and percentage, or median and range or mean and standard deviation where appropriate. Differences between groups were explored using the t-test or Mann-Whitney U-test, where appropriate. A p-value less than 0.05 was considered significant. All analyses were made using the statistical program MedCalc (MedCalc Software bvba, Ostend, Belgium).

\section{Results}

In this study, FF from the leading follicles of 198 women undergoing IVF was analyzed. On average, we received 11.2 oocytes in the PCOS group, 6.5 oocytes in the endometriosis group, nine oocytes in the TFI group and 9.3 oocytes in the healthy control group. The pregnancy rate was highest in the healthy group (60\%), followed by the TFI group (48.8\%); and in the PCOS and endometriosis groups, the pregnancy rate was 39.5 and 38.9, respectively. Based on the manufacturer's reference range for the dROM test, we can indicate our endometriosis and PCOS patients as a group with serious oxidative stress (401-500 Carr.U.). Patients with TFI were in moderate oxidative stress range (341-400 Carr.U.). Healthy patients were in a border-line condition group (300-320 Carr.U.).

Reference values for the PAT test place endometriosis and healthy patients in a group with normal antioxidant values, PCOS patients had slightly elevated antioxidants; only TFI patients had very high results.

The median FF dROM was significantly higher in the endometriosis group (422 Carr.U.) compared with the cases in the control group (283 Carr.U., $\mathrm{p}<0.0001$ ). Median FF dROM results were also substantially higher in the PCOS group (414 Carr.U.) compared with those in the control group (283 Carr.U., $\mathrm{p}<0.0001$ ). There was a significant difference between the median FF dROM test results in the TFI group (375 Carr.U.) compared with those with the control group (283 Carr.U., p < 0.0001).

Patients with endometriosis were associated with significantly higher levels of FF PAT (2751.5 U.Cor), compared with the cases in the control group (2365 U.Cor, $\mathrm{p}=0.0020$ ). The median FF PAT was higher in the PCOS group (2618 U.Cor) compared with the cases in the control group (2346 U.Cor, $\mathrm{p}<0.0004)$. There was also a major difference between the mean FF PAT test results in the TFI group (2954.7 U.Cor) compared with those in the control group (2365 U.Cor, $\mathrm{p}=0.0013$ ).

To evaluate overall oxidative stress, we also calculated the ratio between $\mathrm{dROM}$ and the PAT test. We found a significant difference between the endometriosis group (0.150) and the PCOS group (0.149) of patients compared to the control group, in which the median ratio was 0.120 $(\mathrm{p}=0.0076, \mathrm{p}=0.0049$, respectively). Between the TFI group (0.131) and the healthy group, there were no significant differences in the oxidative stress ratio $(0.120$; $p=0.4933$ ), but the ratio was slightly higher in the TFI group. The results are shown in Table 3 and Figure 1.

Table 3: Results of oxidative stress markers: $\mathrm{dROM}$ and PAT in the follicular fluid and their ratio.

\begin{tabular}{|c|c|c|c|}
\hline & dROM (Carr.U.) & PAT (U.Cor) & dROM/PAT \\
\hline \multicolumn{4}{|c|}{ Endometriosis } \\
\hline Average & 456.6 & $2751.5(p=0.0020)$ & 0.1674 \\
\hline Median & $422(p<0.0001)$ & 2584 & $0.150(p=0.0076)$ \\
\hline \multicolumn{4}{|l|}{ PCOS } \\
\hline Average & 418.4 & 3075.5 & 0.1479 \\
\hline Median & $414(p<0.0001)$ & $2618(p=0.0004)$ & $0.149(p=0.0049)$ \\
\hline \multicolumn{4}{|c|}{ Tubal factor infertility } \\
\hline Average & 392 & $2954.7(p=0.0013)$ & 0.1165 \\
\hline Median & $375(p<0.0001)$ & 2956 & $0.131(p=0.4933)$ \\
\hline \multicolumn{4}{|c|}{ Healthy control } \\
\hline Average & 302.6 & 2365 & 0.1302 \\
\hline Median & 283 & 2346 & 0.120 \\
\hline
\end{tabular}




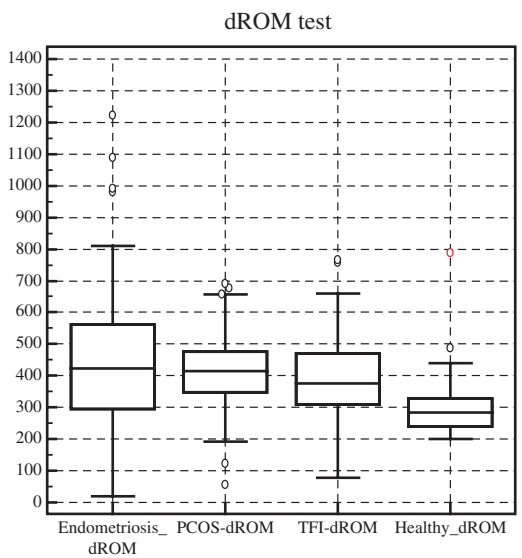

$\mathrm{dROM}$
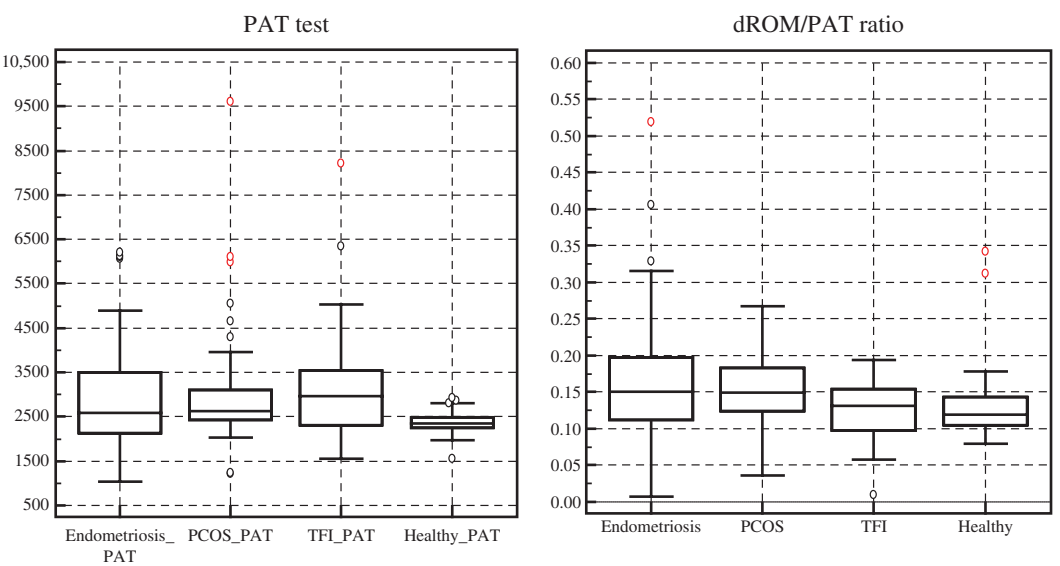

Figure 1: The graphical representation of statistical data.

Average or median concentrations (where appropriate) of both oxidative stress markers: dROM and PAT in the FF were higher in all three groups of patients (PCOS, endometriosis and tubal factor infertility) compared with the healthy control group and differences were statistically significant. The ratio between dROM and PAT was significantly higher in the endometriosis and PCOS groups, and there were no significant differences between the tubal factor infertility and healthy groups.

\section{Discussion}

The results of this study demonstrated that the FF of women with PCOS, endometriosis and tubal diseases has significantly higher levels of the oxidative stress marker $\mathrm{dROM}$ and a higher level of PAT when compared with the women in the control group.

The dROMs test measures the hydroperoxides, which represent the main oxidized molecules in the organism and the more efficient expression of oxidative stress. Markers of peroxidation were measured in the FFs of women attending an IVF to assess the pro-oxidative status. The dROMs test modifications (expressed in Carr.U.) are more advanced than those based on the oxidation of terminal reactive molecules [such as thiobarbituric acid reactive substances (TBARS), malonyldialdehyde (MDA), 4-hydroxynonenal] and also include specific components such as isoprostanes, oxidized DNA or carbonylated proteins that taken singularly may reflect only an incomplete condition of OS. Because of its correlation with the isoprostanes and $\mathrm{C}$-reactive protein (hsCRP), the dROMs test is ideal for determining the redox inflammatory condition (RI) that is the precocious stage of many acute illnesses or a reliable index of permanence for many chronic diseases (such as cardiovascular, pulmonary and metabolic).

It has been observed that the dROMs test serves as a suitable measure for early detection of an imbalanced state like RI that can be a prelude to a general pathological condition [23]. A result showing a higher level of $\mathrm{RS}$ in $\mathrm{FF}$ is in accordance with several previous studies that reported the elevated ROS level marker in the FF of women with PCOS [31] and endometriosis [32-34]. FF makes up the actual environment of the mature oocyte before fertilization and may influence IVF outcome parameters such as fertilization, embryo cleavage and pregnancy rates [35]. In addition to granulosa cells, growth factors and steroid hormones, this environment contains leukocytes, cytokines and macrophages - which can all produce ROS. Hence, ROS may be produced by either the environment or the impaired metabolism of the oocyte, or both [36]. Some studies have already shown that oxidative stress in FF is directly associated with poor IVF pregnancy outcome [32,37,38]. Our results were comparable and also show the link between oxidative stress and the outcome of IVF. The pregnancy rate was expected to be the highest in the healthy group, followed by the TFI group. Our pregnancy rate was approximately $20 \%$ worse for the PCOS and endometriosis groups compared with the healthy patients.

Abnormally high concentrations of free radicals disrupt the balance between oxidants and antioxidants, promoting oxidative stress conditions [39]. In order to protect the follicles from oxidative insult, $\mathrm{FF}$ is naturally provided with an efficient antioxidant system [40]. Our results show significantly higher concentrations of antioxidant in FF in women with endometriosis, PCOS and TFI, compared with those of the healthy control group. These observations are in accordance with the findings of Sabatini and colleagues [41] that higher levels of superoxide dismutase activity were present in fluid from follicles with oocytes that did not fertilize compared with FF with oocytes that fertilized. Our observations also agree with the study by Singh and colleagues [32], who did not 
find important differences in the levels of total antioxidant capacity (TAC), enzymatic antioxidants and vitamins between the non-pregnant and pregnant groups with endometriosis and TFI. They suggested that these molecules may be effective in quenching excessive generation of free radicals, but they do not seem to play a contributory role in achieving successful pregnancy following IVF. This is an important observation in view of the fact that several groups have the opinion that antioxidants, mineral and vitamin supplementation may considerably improve the IVF success rate $[42,43]$.

However, our study conflicts with the results of some other studies [8, 36, 44], which observed that lower levels of antioxidants predict decreased fertilization potential. Inconsistent findings in oxidative stress among investigators are not unusual. The reasons for this discrepancy were not clear. It may reflect methodological differences. A chemical and unspecific standard reaction between iron (III) and thiocyanate has been used extensively as a measure of the antioxidant capacity of fluids and plasma [23]. However, phosphate content in the samples can interfere with the reaction, generating false-positive results. This is why the PAT test contains in its solution a particular zirconium salt that binds phosphates; the antioxidant content will be proportional to the "real" capacity of plasma to reduce $\mathrm{Fe}^{3+}$ and decolor the iron-thiocyanate complex. Furthermore, other studies sometimes also choose a control group, which included women with mechanical causes of infertility. These factors complicate comparison of our results with those of previous research.

It is important to know that endogenous antioxidants work as one big system that combines its main constituents to maintain redox balance in the body. When ROS levels rise and threaten the homeostatic processes of the human body, endogenous antioxidants are activated. The majority of them are expressed when some factors (such as factor Nrf2) are activated. For instance, an estradiol is an antioxidant in cells because it lowers peroxide production by mitochondria [45] and the development and growth of endometriotic lesions are estrogen-dependent [46]. However, endogenous antioxidants also work together with exogenous antioxidants from diet to decrease levels of ROS [47].

Oxidative stress is defined as a disequilibrium between the production and neutralization of ROS, which may result from excess ROS production. Based on this definition we decided to calculate the ratio between $\mathrm{dROM}$ and the PAT test. Our study shows that oxidative stress could be a predictable factor for successful pregnancy. Both tests showed a higher rate of ROS and antioxidants in FF in groups with PCOS and in the endometriosis group.
The ratio between pro-oxidants and antioxidants represents the overall oxidative stress milieu and it seems to be a significantly better predictor of achieving pregnancy than the ROS level or antioxidants level alone. The ratio is, however, significantly higher in both disease groups. It is therefore assumed that a different diagnosis can also be affected by a higher degree of oxidative stress. Both PCOS and endometriosis are complex endocrine disorders. The disrupted hormonal balance, of course, affects the metabolism, which can also be reflected in the broken balance of oxidants. This can also happen in the follicular fluid and certainly contributes to the reduced ability to fertilization. There was no significant difference in the dROM/PAT ratio between the TFI group and the healthy group. In principle, patients with TFI diagnosis have only physical reasons for infertility; otherwise, they are healthy patients. However, our results show that patients with TFI had an elevated degree of oxidative stress, which is why we did not take them as a healthy group as some researchers usually did. The pregnancy rate is also lower in the TFI group than in our healthy control group. We assumed that the removal of the fallopian tubes in these patients solved only a secondary problem, but not a cause, which could have been due to defective metabolism or even endocrine disruption. It seems that a composite dROM/PAT ratio may be more strongly correlated with infertility than $\mathrm{dROM}$ or PAT alone. Therefore, the dROM/PAT ratio may serve as an important measure in identifying those patients who are more likely to achieve a pregnancy over a period of time.

The present data do not support the modification of any current clinical protocol regarding the assessment of the systemic oxidative balance in infertile women. However, the present paper intends to trigger discussion on the need for performing longitudinal studies to investigate the profile of oxidative stress markers during treatment for assisted reproduction and their potential impact on the results of such treatments. If a negative impact of systemic OS on reproductive results is confirmed, it will be necessary to define the prognostic markers to be assessed, and to conduct well-designed trials investigating the roles of different antioxidants in women with a diagnosis of systemic oxidative stress.

\section{Conclusions}

Oxidative stress seems to be an important causative factor in the etiology of many diseases including PCOS, endometriosis and TFI. It is therefore necessary to conduct further research to determine the optimal levels in $\mathrm{FF}$ that allows successful IVF to be carried out. In future work, we plan 
to measure OS markers in maternal sera and FF to estimate the circulating or intraovarian origin of the relevant factors.

Acknowledgments: The authors would like to thank all women in IVF programme who agreed to participate in the study, and to Vera Troha who processed samples in lab.

Author contributions: All the authors have accepted responsibility for the entire content of this submitted manuscript and approved submission.

Research funding: This study was financed by the Ministry of Science and Education through Young Researchers programme.

Employment or leadership: None declared. Honorarium: None declared.

Competing interests: The funding organization(s) played no role in the study design; in the collection, analysis, and interpretation of data; in the writing of the report; or in the decision to submit the report for publication.

\section{References}

1. Ghiselli A, Serafini M, Natella F, Scaccini C. Total antioxidant capacity as a tool to assess redox status: critical view and experimental data. Free Radic BiolMed 2000;29:1106-14.

2. Halliwell B, Cross CE. Oxygen-derived species: their relation to human disease and environmental stress. Environ Health Perspect 1994;102:5-12.

3. Davies KJ. Oxidative stress: the paradox of aerobic life. Biochem Soc Symp 1995;61:1-31.

4. Lushchak VI. Glutathione homeostasis and functions: potential targets for medical interventions. J Amino Acids 2012;2012: $1-26$.

5. Sies $\mathrm{H}$. Role of metabolic $\mathrm{H}_{2} \mathrm{O}_{2}$ generation: redox signaling and oxidative stress. J Biol Chem 2014;289:8735-41.

6. Dumesic DA, Meldrum DR, Katz-Jaffe MG, Krisher RL, Schoolcraft WB. Oocyte environment: follicular fluid and cumulus cells are critical for oocyte health. Fertil Steril 2015;103:303-16.

7. Celik E, Celik O, Kumbak B, Yilmaz E, Turkcuoglu I, Simsek Y, et al. A comparative study on oxidative and antioxidative markers of serum and follicular fluid in $\mathrm{GnRH}$ agonist and antagonist cycles. J Assist Reprod Genet 2012;29:1175-83.

8. Oyawoye O, Abdel Gadir A, Garner A, Constantinovici N, Perrett C, Hardiman P. Antioxidants and reactive oxygen species in follicular fluid of women undergoing IVF: relationship to outcome. Hum Reprod 2003;18:2270-4.

9. Revelli A, Delle Piane L, Casano S, Molinari E, Massobrio M, Rinaudo P. Follicular fluid content and oocyte quality: from single biochemical markers to metabolomics. Reprod Biol Endocrinol 2009;7:40.

10. Sciarra J. Infertility: an international health problem. Int J Gynecol Obstet 1994;46:155-63.

11. WHO. Infertility: a tabulation of available data on prevalence of primary and secondary infertility. Geneva: World Health Organization, 1991.
12. Okonofua FE. The case against new reproductive technologies in developing countries. Br J Obstet Gynaecol 1996;103:957-62.

13. Olive DL, Schwartz LB. Endometriosis. N Engl J Med 1993;328:1759-69.

14. Giudice LC, Kao LC. Endometriosis. Lancet 2004;364:1789-99.

15. Bulun SE. Endometriosis. N Engl J Med 2009;360:268-79.

16. Brosens I, Benagiano G. Endometriosis, a modern syndrome. Indian J Med Res 2011;133:581-93.

17. Rižner TL. Estrogen metabolism and action in endometriosis. Mol Cell Endocrinol 2009;307:8-18.

18. Sourial S, Tempest N, Hapangama DK. Theories on the pathogenesis of endometriosis. Int J Reprod Med 2014;2014:1-9.

19. Sampson JA. Peritoneal endometriosis due to the menstrual dissemination of endometrial tissue into the peritoneal cavity. Am J Obstet Gynecol 1927;14:422-69.

20. Berkkanoglu M, Arici A. Immunology and endometriosis. Am J Reprod Immunol 2003;50:48-59.

21. Akande VA. Tubal disease: towards a classification. Reprod Biomed Online 2007;15:369-75.

22. Khalaf Y. Tubal subfertility. Br Med I 2003;327:610-3.

23. Benzie IF, Strain JJ. The ferric reducing ability of plasma (FRAP) as a measure of "antioxidant power": the FRAP assay. Anal Biochem 1996;239:70-6.

24. Halliwell B. Free radicals, antioxidants, and human disease: curiosity, cause, or consequence? Lancet 1994;344:721-4.

25. Verde V, Fogliano V, Ritieni A, Maiani G, Morisco F, Caporaso N. Use of N,N-dimethyl-p-phenylenediamine to evaluate the oxidative status of human plasma. Free Radic Res 2002;36:869-73.

26. Alberti A, Bolognini L, Macciantelli D, Caratelli M. The radical cation of $\mathrm{N}, \mathrm{N}$-diethyl-para-phenylendiamine: a possible indicator of oxidative stress in biological samples. Res Chem Intermed 2000;26:253-67.

27. Cesarone MR, Belcaro G, Carratelli M, Cornelli U, De Sanctis MT, Incandela L, et al. A simple test to monitor oxidative stress. Int Angiol 1999;18:127-30.

28. Cavalleri A, Colombo C, Venturelli E, Miceli R, Mariani L, Cornelli $U$, et al. Evaluation of reactive oxygen metabolites in frozen serum samples. Effect of storage and repeated thawing. Int J Biol Markers 2004;19:250-3.

29. Pasquini A, Luchetti E, Marchetti V, Cardini G, Iorio EL. Analytical performances of d-ROMs test and BAP test in canine plasma. Definition of the normal range in healthy Labrador dogs. Vet Res Commun 2008;32:137-43.

30. Cornelli U, Belcaro G, Finco A. The oxidative stress balance measured in humans with different markers, following a single oral antioxidants supplementation or a diet poor of antioxidants. J Cosmet Dermatological Sci Appl 2011;1:64-70.

31. Turan V, Sezer ED, Zeybek B, Sendag F. Infertility and the presence of insulin resistance are associated with increased oxidative stress in young non-obese turkish women with polycystic ovary syndrome. J Pediatr Adolesc Gynecol 2015;28:119-23.

32. Singh AK, Chattopadhyay R, Chakravarty B, Chaudhury K. Markers of oxidative stress in follicular fluid of women with endometriosis and tubal infertility undergoing IVF. Reprod Toxicol 2013;42:116-24.

33. Liu F, He L, Liu Y, Shi Y, Du H. The expression and role of oxidative stress markers in the serum and follicular fluid of patients with endometriosis. Clin Exp Obstet Gynecol 2013;40:372-6. 
34. Prieto L, Quesada JF, Cambero O, Pacheco A, Pellicer A, Codoceo $\mathrm{R}$, et al. Analysis of follicular fluid and serum markers of oxidative stress in women with infertility related to endometriosis. Fertil Steril 2012;98:126-30.

35. Agarwal A, Saleh RA, Bedaiwy MA. Role of reactive oxygen species in the pathophysiology of human reproduction. Fertil Steril 2003;79:829-43.

36. Das S. Reactive oxygen species level in follicular fluid - embryo quality marker in IVF? Hum Reprod 2006;21:2403-7.

37. Borowiecka M, Wojsiat J, Polac I, Radwan M, Radwan P, Zbikowska HM. Oxidative stress markers in follicular fluid of women undergoing in vitro fertilization and embryo transfer. Syst Biol Reprod Med 2012;58:301-5.

38. Bedaiwy MA, El-Nashar SA, Goldberg JM, Sharma R, Mascha EJ, Arrigain S, et al. Effect of follicular fluid oxidative stress parameters on intracytoplasmic sperm injection outcome. Gynecol Endocrinol 2012;28:51-5.

39. Agarwal A, Gupta S, Sharma RK. Role of oxidative stress in female reproduction. Reprod Biol Endocrinol 2005;3:28.

40. Agarwal A, Said TM, Bedaiwy MA, Banerjee J, Alvarez JG. Oxidative stress in an assisted reproductive techniques setting. Fertil Steril 2006;86:503-12.

41. Sabatini L, Wilson C, Lower A, Al-Shawaf T, Grudzinskas JG. Superoxide dismutase activity in human follicular fluid after controlled ovarian hyperstimulation in women undergoing in vitro fertilization. Fertil Steril 1999;72:1027-34.

42. Özkaya MO, Nazıroğlu M. Multivitamin and mineral supplementation modulates oxidative stress and antioxidant vitamin levels in serum and follicular fluid of women undergoing in vitro fertilization. Fertil Steril 2010;94:2465-6.

43. Özkaya MO, Nazıroğlu M, Barak C, Berkkanoglu M. Effects of multivitamin/mineral supplementation on trace element levels in serum and follicular fluid of women undergoing in vitro fertilization (IVF). Biol Trace Elem Res 2011;139:1-9.

44. Paszkowski T, Traub Al, Robinson SY, McMaster D. Selenium dependent glutathione peroxidase activity in human follicular fluid. Clin Chim Acta 1995;236:173-80.

45. Borrás C, Gambini J, López-Grueso R, Pallardó FV, Viña J. Direct antioxidant and protective effect of estradiol on isolated mitochondria. Biochim Biophys Acta - Mol Basis Dis 2010;1802:205-11.

46. Bulun SE, Zeitoun KM, Takayama K, Sasano H. Estrogen biosynthesis in endometriosis: molecular basis and clinical relevance. J Mol Endocrinol 2000;25:35-42.

47. Fregoso Aguilar TA, Hernandez Navarro BC, Mendoza Perez JA. Endogenous antioxidants: a review of their role in oxidative stress. In: Morales-Gonzalez JA, Morales-Gonzalez A, Madrigal-Santillan EO, editors. A master regulator of oxidative stress - the transcription factor Nrf2. London: InTech, 2016. doi:10.5772/65715. 